\title{
The effects of payoffs and prior probabilities on indices of performance and cutoff location in recognition memory
}

\author{
ALICE F. HEALY \\ Yale University, New Haven, Connecticut 06520 \\ and Haskins Laboratories, New Haven, Connecticut 06511 \\ and \\ MICHAEL KUBOVY \\ Yale University, New Haven, Connecticut 06520
}

\begin{abstract}
We compared the effects of payoffs and prior probability levels on indices of performance and cutoff location in recognition memory. The performance indices considered were $d^{\prime}$ from signal-detection theory and two corrections for guessing from simple threshold models. Shifts in cutoff location of comparable size were found for changes in priors and payoffs. The existence of a memory load and the inclusion of feedback did not affect the extent of shifts in cutoff location. Priors affected the corrections for guessing but did not affect $d^{\prime}$. These results indicate that $d^{\prime}$ remains the preferred index of performance and that the corrections for guessing are not criterion-free measures. The problem of finding an index of recognition performance appropriate for comparing recall and recognition is discussed, as are alternative explanations for conservative cutoff placement.
\end{abstract}

This paper deals with two issues: The first issue concerns the effects of payoffs and prior probability levels on indices of recognition memory performance, or memory storage. The second issue, which provided the motivation for the present study, concerns the effects of these variables on cutoff location.

\section{Indices of Performance}

A criterion-free index of performance is necessary when one is dealing with a recognition memory task. In a typical recognition memory task, subjects first study a group of items. Then their memory is tested by asking them to classify each of a series of items, which includes the studied ("old") items and distractor

This research was supported in part by PHS Grants MH26573 and RR07015 and NSF Grant BNS 77-00077 to Yale University, and by NICHD Grant HD01994 and BRSG Grant RR05596 to Haskins Laboratories. A. F. Healy was supported by a Junior Faculty Fellowship from Yale University during the preparation of the manuscript. We are indebted to Randy Smith, who helped with the computer programming of the experiment, Wendy Brown, who helped design the experiment and analyze the data, Oren Patashnik, who helped with the computer programming and helped design, conduct, and analyze the experiment, and Loretta Polka, who helped analyze the data. We also thank B. B. Murdock, Jr., R. G. Crowder, and an anonymous reviewer for careful review of an earlier version of this manuscript and many helpful suggestions. In addition, we are most grateful to W. K. Estes for suggesting the comparison of the three performance indices. Requests for reprints should be sent to Alice F. Healy, Department of Psychology, Box 11A Yale Station, Yale University, New Haven, Connecticut 06520. ("new") items, as old (by responding "yes") or new (by responding "no"). In this situation, subjects are free to vary their tendency to call a given item old or new. They may adopt a strict criterion and say "yes" only when they are certain that they recognize the item, or they may adopt a lenient criterion and say "yes" more frequently. Hence, the hit rate (HR), $p$ ("yes"|old), is a poor index of performance because it is contaminated by the strictness of the subject's criterion; it is not criterion free. Other indices, such as the false-alarm rate (FAR) and the proportion of correct responses, suffer from the same problem. In order to solve this problem, a number of criterion-free indices of performance have been proposed, including two corrections for guessing based on simple threshold models of recognition memory. Both of these models have been called "high-threshold" models (see, e.g., Murdock, 1974), but, actually, one of the models includes two thresholds, as will be discussed below. Our discussion of these models follows that of Egan (Note 1), where a fuller description is available. The corrections for guessing are derived from the following equations, which embody the assumptions of these simple threshold models: $\mathrm{HR}=[\mathrm{p}+(1-\mathrm{p}) \mathrm{g}]$ and FAR $=(1-\mathrm{r}) \mathrm{g}$, where $p$ is the theoretical (as opposed to observed) probability that an old item will be recognized as such, $r$ is the theoretical probability that a new item will be recognized as such, and $\mathrm{g}$ is the guessing rate. In effect, three states are assumed: one in which old items are recognized as such, a second in which new items are 
recognized as such, and an intermediate state in which the subject must guess. Two thresholds separate these three states. Assuming that $r=0$, we obtain a two-state single-threshold model from which we derive the following index of performance: $p_{1}=(H R-F A R) /(1-F A R)$. Similarly, assuming that $r=p$, we obtain a three-state double-threshold model from which we derive the following index of performance: $p_{2}=H R-F A R$. These indices of performance are especially valuable because they estimate the amount of information stored in memory and they are comparable (at least at first blush) to the standard measure of recall performance, proportion of correctly recalled items. ${ }^{1}$

Another criterion-free index of recognition performance is $d^{\prime}$, derived from the theory of signal detection (TSD). The most common comparative empirical tests of TSD and simple threshold theories of recognition memory have been based on qualitative examination of the shape of the memory operating characteristic (MOC), the plot of HR by FAR. According to the simple threshold theories, the MOC should be a single straight line, whereas, according to TSD, it should be bow shaped. The data (e.g., Green \& Moses, 1966; Murdock, 1965; Egan, Note 1) support TSD (or more elaborate threshold theories, such as Krantz, 1969; Luce, 1963). Nevertheless, TSD's index of performance, $\mathrm{d}^{\prime}$, has not been used in all situations, because, unlike the above-mentioned indices of performance based on the simple threshold models, $\mathrm{d}^{\prime}$ is not comparable to indices of recall performance. (A Type II $\mathrm{d}^{\prime}$ can be computed for a recall task, but it would not be comparable to the Type $I \mathrm{~d}^{\prime}$ used in recognition and would not provide an estimate of memory storage. See Healy and Jones, 1973, for a discussion of the Type II TSD analysis as applied to recall.) In studies where a comparison of recall and recognition is at issue and the recognition test employed involves a yes-no procedure (e.g., Anderson, 1976, p. 421; Estes \& DaPolito, 1967; Kintsch, 1970; Tulving \& Thomson, 1973), $d^{\prime}$ is not a useful index. The question remains whether $p_{1}$ and $p_{2}$ are serviceable for such situations. We should stress, however, that the present study was not motivated by an approval for the use of these guessing corrections but, rather, by the very fact that investigators of recognition memory have continued to use these corrections in a number of circumstances despite the evidence against them from MOC curves.

In this paper, we attempt to determine whether $p_{1}$ and $p_{2}$ are adequate indices of recognition performance. Specifically, we aim to determine whether $p_{1}$ and $p_{2}$ are criterion free or whether, instead, they vary as a function of payoffs and prior probabilities. If they do vary with payoffs and priors, they would be in no way preferable to HR, FAR, or proportion of correct responses, the measures of performance we rejected above. In addition to $p_{1}$ and $p_{2}$, we consider, for comparison purposes, the effects of changes in payoffs and priors on $\mathrm{d}^{\prime}$.
We should point out that tests of the invariance of the different performance indices in the face of changes in payoffs and priors are not independent of tests based on MOC curves. If data points for different priors and payoffs fall on the MOC predicted by a given theory, then the index of performance for that theory must be invariant across such changes in priors and payoffs. Conversely, if data points do not lie on the MOC predicted by the theory, then the index for that theory will not be invariant. Hence, from the previous qualitative tests, we expect to find $\mathrm{d}^{\prime}$ to be more stable than $p_{1}$ and $p_{2}$ in the present comparison. The present quantitative tests should reveal the extent to which the various performance indices deviate significantly from invariance. Also, the present tests should be in some sense more straightforward than tests based on MOC curves, since the present tests should reveal directly the extent to which the alternative "criterion-free" indices are in fact influenced by changes in the criterion.

\section{Cutoff Location}

Although early studies in auditory signal detection (e.g., Swets, Tanner, \& Birdsall, 1961) have shown that beta (TSD's index of the location of the cutoff point along the decision axis) is affected by prior probabilities and payoffs, several recent studies of recognition memory have demonstrated that beta is insensitive to prior probabilities (Healy \& Jones, 1975; Murdock, 1974). Murdock (1974) has also reported an unpublished recognition memory study by Wells in which prior probability did affect beta, but he pointed out that the changes in prior probability level in that study were confounded with other changes in the experimental situation. Healy and Kubovy (1977) compared a recognition memory task, in which subjects judged whether a given five-digit number was old or new, to a numerical decision task, in which subjects judged whether a given five-digit number represented the height of a man or a woman. Subjects shifted beta with changes in prior probabilities in the direction specified by TSD in the numerical decision task but not in the recognition memory task.

There are a number of possible explanations for the subjects' relative insensitivity to prior probabilities in recognition memory. Healy and Kubovy (1977) discussed some of these explanations. In the present study, we were primarily concerned with a single explanation, which we shall refer to as the "memoryload hypothesis": The recognition memory task may impose memory requirements that are incompatible with the effective use of prior probability information. In particular, a subject may find it difficult to keep track of the number of old items occurring on the test if he also has to hold in memory the study items.

We tested the memory-load hypothesis in three different ways. First, we compared the standard recognition memory situation ("standard" condition), in which subjects were told how many old and new items to 
expect and were given feedback on correct responses, to two other conditions that differed in terms of the memory requirements necessary for subjects to keep track of the number of old items occurring on the test. At one extreme, we provided subjects, in addition to the information given in the standard condition, a running tally of the number of old and new items remaining on the test ("tally" condition), and, at the other extreme, we provided subjects with no feedback at all concerning whether the tested items were old or new ("no-feedback" condition). According to the memoryload hypothesis, subjects should be most serisitive to priors in the tally condition, less sensitive in the standard condition, and least sensitive in the no-feedback condition. Second, we compared the standard recognition memory task to a task that imposed no memory requirements but was otherwise strictly analogous to the standard recognition memory task ("no-memory" condition). Specifically, in the no-memory condition, subjects were told how many old and new items to expect, without committing any items to memory. Thus, the subjects were compelled to guess the sequence of correct responses, given (1) prior probabilities and (2) feedback on correct responses. (This task is similar to probability learning, except that here subjects are told the prior probabilities.) According to the memoryload hypothesis, subjects should be more sensitive to priors in the no-memory condition than in the standard condition. Third, we compared the standard recognition memory task with changes in prior probabilities to an analogous recognition memory task in which payoffs but not prior probabilities were changed ("payoff" condition). The payoff matrices in the pay off condition led to the same optimal beta levels as the priors in the standard condition. Assuming that the use of payoffs taxes memory less than the use of priors (i.e., subjects need not keep track of the number of old and new items occurring on the test to make effective use of pay offs), according to the memory-load hypothesis, subjects should be more sensitive to changes in payoffs than to changes in priors.

This experiment should have implications about probabilistic categorization decisions in general in addition to its implications about decision making in recognition memory. Although subjects are not totally insensitive to prior probabilities in other situations involving probabilistic categorization decisions, such as in tasks involving signal detection or numerical decision (Kubovy \& Healy, 1977), conservative cutoff placement is found in those situations. In other words, subjects have been found to place their cutoffs at a point that is less extreme than the one that would maximize expected monetary gain. A number of possible explanations for this behavior have been proposed and are summarized in Kubovy (1977) and Kubovy and Healy (in press).

One cause of conservative cutoff placement suggested by Kubovy (1977) is that the subject conforms to the optimal behavior specified by TSD except for a systematic misconception of the shape of the underlying stimulus distributions. Specifically, Kubovy has argued that subjects tend to overestimate likelihood ratios that are greater than one and underestimate likelihood ratios that are less than one, and such false estimations would lead to conservative cutoff placement. This hypothesis would lead one to expect conservative cutoff placement in the recognition memory conditions but not in the nomemory condition, since the subject should essentially ignore the stimulus distributions in the no-memory condition. Finding subjects to be equally conservative in their cutoff placements in the standard and nomemory conditions would provide evidence against this hypothesis.

Other explanations for conservative cutoff placement are specific to the ways in which subjects interpret either prior probabilities or payoffs. These hypotheses would lead one to expect that the extent of conservative cutoff placement would be different for changes in payoffs and priors, because conservative cutoff placement would have different causes in the two situations. For example, Galanter (1974) and Ulehla (1966) have proposed that when payoffs vary, conservative cutoff placement can be explained by the form of the utility function for money. According to this explanation, as in Kubovy's (1977) explanation, the subject essentially conforms to the normative prescriptions except for a miscalculation, in this case a miscalculation of the optimal value of beta specified by the payoff matrix. An analogous explanation proposed by Ulehla (1966) for the case when priors vary is that subjects underestimate high prior probabilities and overestimate low ones, leading to a miscalculation of the optimal value of beta. Another hypothesis that concerns only the situations when priors vary is that conservative cutoff placement could be caused by the subjects' tendency to match the proportions of their "yes" and "no" responses to the prior probabilities (Craig, 1976; Creelman \& Donaldson, 1968; Dorfman, 1969; Parks, 1966; Thomas \& Legge, 1970). According to these three explanations, a correspondence in cutoff shifts for changes in payoffs and priors should occur only by coincidence. The comparison of the standard and payoff conditions of the present experiment will provide a test of these hypotheses.

A class of explanations for conservative cutoff placement that would predict a correspondence between degrees of conservative cutoff placement for payoffs and prior probabilities also includes the assumption of essentially optimal behavior by the subject except for a systematic miscalculation. In this case, the miscalculation does not involve the computation of the optimal value of beta on the basis of the payoffs or the priors. Rather, the miscalculation involves where to place the cutoff on the observation continuum given the correct computation of the optimal value of beta. 
Kubovy's (1977) distribution-misconception explanation, discussed above, falls into this class, but other examples of this type presumably exist as well, although they have not been clearly specified as yet.

\section{METHOD}

\section{Subjects}

Sixty Yale College students participated: Fifty-one received course credit for introductory psychology; the remaining nine received a fee of $\$ 2.50$. In addition, all subjects were paid a bonus of .2 cents per point earned (see below). Ten subjects received a bonus of 50 cents even though they earned less than 250 points. These subjects were not informed of the additional profit until the end of the experiment. There were five conditions with 12 subjects in each: payoff, standard, no feedback, tally, and no memory.

\section{Apparatus}

A Digital Equipment Corporation VT50 terminal, controlled by a Digital Equipment Corporation PDP-11/40 computer, operating under a timesharing system, was used for the display of the stimuli and the collection of the responses.

\section{Design and Procedure}

Each subject was tested for approximately $45 \mathrm{~min}$, being exposed to eight blocks of trials with a rest period after the first four.

Payoff condition. Each block of trials included a study phase and a test phase. During the study phase, the subject saw 20 five-digit numbers to be remembered. Then, during the test phase, the subject responded to 40 five-digit numbers, indicating ("yes" or "no") whether each number had been shown during the study phase. The 40 test items in each block included the 20 study numbers (old items) and 20 distractors (new items). Different sets of numbers were used for each block. ${ }^{2}$

The two payoff matrices shown in Table 1 were employed in this condition. For Matrix 1, optimal beta is 1 , and for Matrix 3, optimal beta is 3 . The entries of these matrices are points, worth .2 cents. Subjects were asked to maximize the number of points earned. Six subjects were assigned to each of two permutations

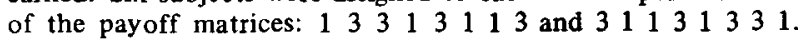

Each block of trials was organized as shown in Figure 1: At the start of each block, the word STUDY was shown on the screen for approximately $3 \mathrm{sec}$. The screen was then blank for $1 \mathrm{sec}$. Next appeared the 20 study items, displayed successively, each for 2 sec, followed by a 1 -sec blank screen. Then the word TEST appeared on the screen for $3 \mathrm{sec}$, followed by a blank screen for $1 \mathrm{sec}$. Then one of the payoff matrices with its label (A or B, see below) appeared on the screen until the subject pressed the RETURN key. The screen was then cleared and the following query was displayed: WHICH MATRIX WAS THAT? (A OR B). The subject was then to press either the A key or the B key, followed by RETURN. If the subject did not respond correctly, the query was repeated. After the subject typed the correct response, the following message was displayed: GET READY: TEST WILL START WHEN RETURN IS PRESSED. After the subject pressed RETURN, the screen was cleared and the 40-item test sequence began. Each test item was presented, with the following message below it: TYPE

Table 1

\begin{tabular}{crrrrr} 
& \multicolumn{2}{c}{ Matrix 1 } & & \multicolumn{2}{c}{ Matrix 3 } \\
\cline { 5 - 6 } \cline { 5 - 6 } & "No" & "Yes" & & "No" & "Yes" \\
\hline Old & -5 & 5 & & -1 & 1 \\
New & 5 & -5 & 3 & -3 \\
\hline
\end{tabular}
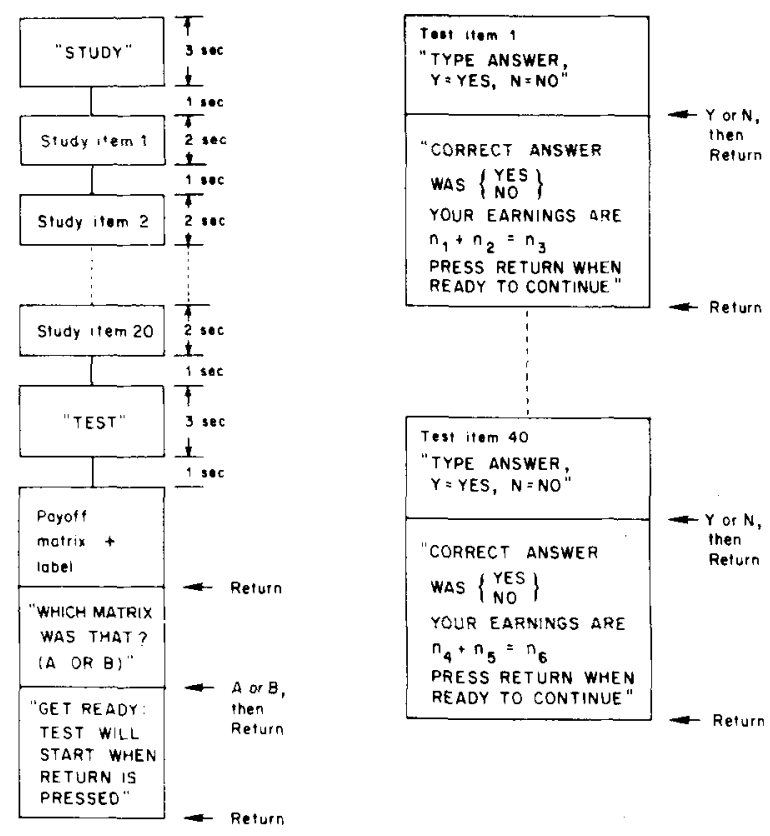

Figure 1. Organization of a block of trials in the payoff condition.

ANSWER, $\mathrm{Y}=\mathrm{YES}, \mathrm{N}=\mathrm{NO}$. If the subject pressed an inappropriate key, the screen displayed the message: INAPPROPRIATE ANSWER, PLEASE TYPE 'Y' OR 'N.' As soon as the subject typed a $\mathrm{Y}$ or an $\mathrm{N}$ and pressed RETURN, feedback was provided in the following form: CORRECT ANSWER WAS YES or CORRECT ANSWER WAS NO, along with a message such as: YOUR EARNINGS ARE $23+3=26$. PRESS RETURN WHEN READY TO CONTINUF. The earnings were given in points. When the subject pressed RETURN, the screen was cleared and the next test item appeared. After the 40th test item and feedback, the second block started. After the completion of the first four blocks, the subject rested for $2 \mathrm{~min}$.

Before the first block, the subjects were given written instructions describing the prior probability information (how many old and new test items would be shown during each test block) and the two payoff matrices and providing the labels for the two matrices (A for Matrix 1 and $B$ for Matrix 3). This information remained visible to the subjects throughout the experiment. In addition, the subjects were given an initial computer-assisted test of their understanding of the prior information and of the two payoff matrices.

Standard condition. This condition was closely matched to the payoff condition. The essential difference was that the symmetric matrix (Matrix 1) was employed for all blocks in the standard condition, whereas two prior probabilities of test items were employed-Prior 1: 20 old items, 20 new items; Prior 3: 10 old items, 30 new items. For Prior 1, optimal beta is 1 , and for Prior 3, optimal beta is 3 . Six subjects were assigned to each of two permutations of prior probabilities: $\begin{array}{lllllllllll}1 & 3 & 3 & 1 & 3 & 1 & 1 & 3\end{array}$ and $\begin{array}{lllllll}3 & 1 & 1 & 3 & 1 & 3 & 3\end{array} 1$. These permutations parallel those for the payoff matrices used in the payoff condition.

Subjects in the standard condition were informed of the prior probabilities for a given block at the start of each test phase and were immediately tested on this information in a similar manner to that used for testing the payoff information in the payoff condition. Subjects were also given an initial computer-assisted test on the two prior probabilities and the payoff matrix. This test was analogous to that employed in the payoff condition. Throughout the standard condition, as in the payoff condition. information about the payoff matrix and the two prior 
probabilities with their labels (A and $B$ ) was visible to the subject.

No-feedback and tally conditions. The no-feedback condition was identical to the standard condition except that the subject was told neither the correct answer nor how many points he had earned thus far. On the other hand, the tally condition was identical to the standard condition except that before each test item appeared on the screen, the subject was told how many new items and how many old items remained in the current test phase. Specifically, $1 \mathrm{sec}$ before each test item appeared, the following message was displayed: IN THIS TEST BLOCK THERE REMAIN (INCLUDING PRESENT ITEM): OLD ITEMS $-X$ and NEW ITEMS-Y. $X$ and $Y$ stand for the numbers of old and new items remaining, respectively. This message remained on the screen with the test item until the subject responded.

No-memory condition. This condition was identical to the standard condition with one exception: No numbers were shown during the study phase. Instead, each five-digit number was replaced by five dashes. To explain this peculiar procedure, the following instructions were added to those of the standard condition: "The above instructions describe a task (call it the memory task) which differs from your task in one important respect-namely, in what will be shown during the study phase. You, as a member of a special control group, will NOT be shown any numbers during the study phase. Instead, where each 5-digit number would have appeared, you will see 5 dashes. Aside from this difference, your task is exactly the same as the memory task. You will see the same 40 test items in each section as will people given the memory task, and the correct answer to a given test item in your task is the same as the correct answer to that item in the memory task. The only effective difference between your task and the memory task is that you will have no memory information on which to base your response."

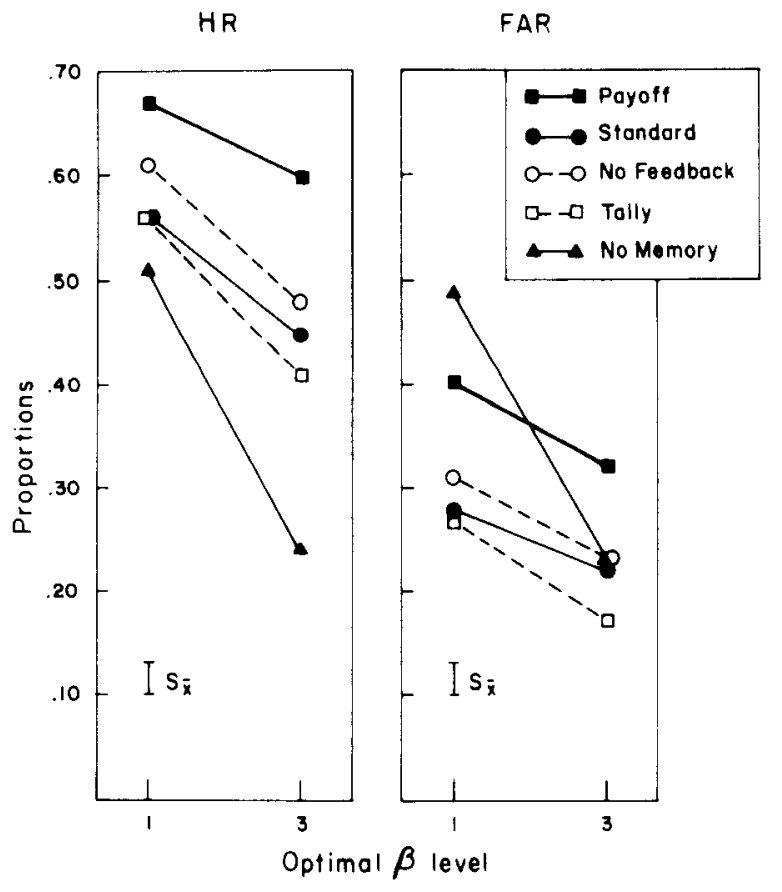

Figure 2. Mean values of HR (left panel) and FAR (right panel) as a function of condition and optimal beta level. The standard error of the means for each index, as determined by an analysis of variance, is indicated at the bottom of the appropriate panel.
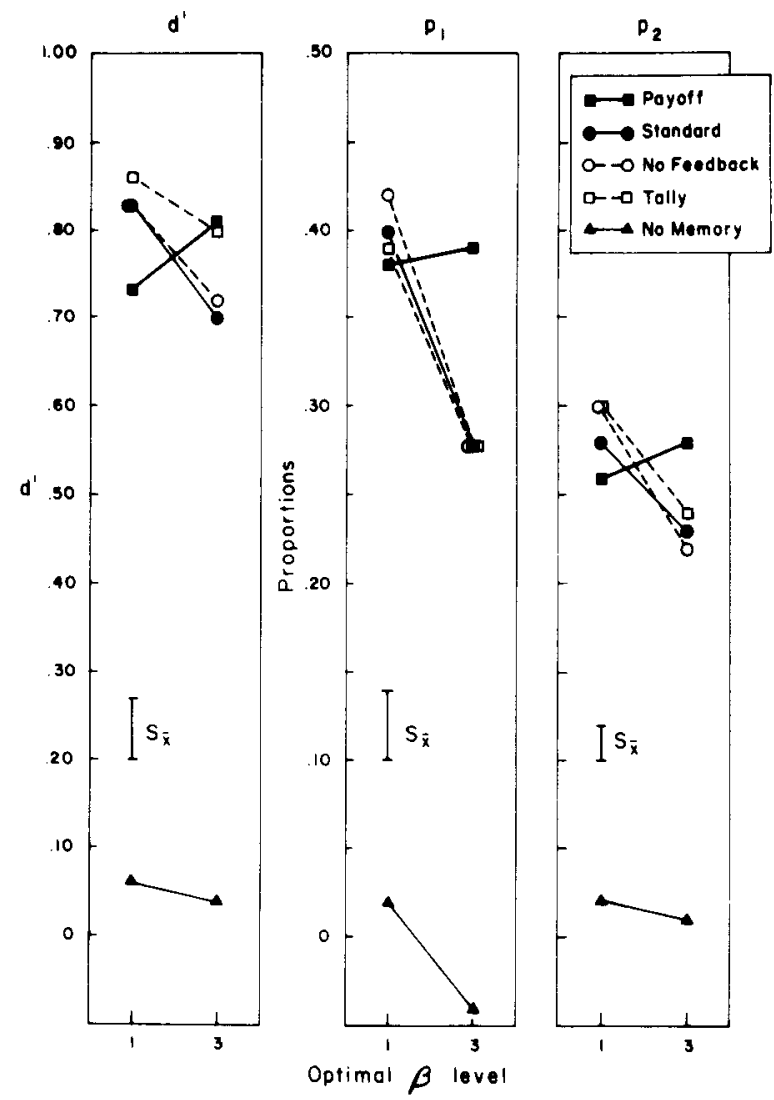

Figure 3. Mean values of $d^{\prime}$ (left panel), $p_{1}$ (center panel), and $p_{2}$ (right panel) as a function of condition and optimal beta level. The standard error of the means for each index, as determined by an analysis of variance, is indicated at the bottom of the appropriate panel. Note that because performance was essentially at chance in the no-memory condition, FAR sometimes exceeded $H R$, yielding negative values of $p_{1}$ and $p_{2}$.

\section{RESULTS AND DISCUSSION}

\section{Indices of Performance}

Effects of condition. HR, FAR, and the three performance indices were computed for each test block. For two subject blocks, the FAR was 1; for those blocks, $p_{1}$, which would otherwise be undefined, was set equal to 0 . Here and in all succeeding computations, the standard score for a HR or a FAR with the value exactly 0 or 1 was taken to be +2 or -2 , respectively. Figures 2 and 3 present the mean values of HR, FAR, $d^{\prime}, p_{1}$, and $\mathrm{p}_{2}$ averaged across subjects and blocks as a function of optimal beta level and condition. In these figures and in the subsequent one, we have averaged across blocks with the same optimal beta level despite occasional significant main effects and interactions involving the factor of blocks, since these interactions were difficult to interpret. Performance in this experiment was generally at the level expected: The recognition memory conditions yielded values of $\mathrm{d}^{\prime}$ close to those reported by Healy and Kubovy (1977), whereas the no-memory condition yielded a value of $d^{\prime}$ close to 0 . 
Performance was roughly equal in all conditions but the no-memory condition. For each of the three performance indices, three sets of analyses of variance were conducted comparing the standard condition with each of the three other recognition memory conditions. For $p_{1}$ and $p_{2}$, analyses were performed both on the raw values and on the values transformed by the folded root (froot) transformation suggested by Mosteller and Tukey (1977): froot(y) $=\sqrt{2 y}-\sqrt{2(1-y)}$. (Although theoretical values of $p_{1}$ and $p_{2}$ are limited to a range between 0 and 1 , negative values do in fact occur whenever FAR $>$ HR. In those cases, the negative values were truncared to 0 before applying the froot transformation. Twenty-five of the 384 subject blocks in the four memory conditions and 37 of the 96 subject blocks in the no-memory condition were truncated.) In no case was there a significant main effect of condition or a significant interaction of condition and optimal beta level. The lack of difference between performance levels in the recognition memory conditions is quite noteworthy. It suggests that level of performance in recognition memory is not influenced by large changes in the amount of feedback provided.

In order to determine whether subjects did pay attention to the extra feedback information provided to them in the tally condition, the numbers of errors on the last trial of each test block were computed. Subjects in the tally condition should essentially never make an error on the last trial of a test block, since they are told how many old and new items remain in each test block before each test item is presented. In fact, out of 96 subject blocks in the tally condition, there were only two errors on the last trial, and each of those was in the first block of a session. In contrast, there were 45 out of 96 subject blocks with errors in the no-feedback condition and 43 out of 96 in the standard condition. This result demonstrates that, although the extra feedback information given in the tally condition did not improve overall performance levels, it was not ignored by the subjects, at least on the last trial.

Effects of optimal beta level. Of critical interest are the effects of optimal beta level (priors and payoffs) on HR, FAR, and the three indices of performance.

In the overall analyses including all conditions, the effect of optimal beta level was significant for HR $[\mathrm{F}(1,50)=64.5, \quad \mathrm{MSe}=.05, \quad 1 / 2 \mathrm{p}<.001]$ and $\mathrm{FAR}$ $[\mathrm{F}(1,50)=46.0, \mathrm{MSe}=.04,1 / 2 \mathrm{p}<.001]$. (Here and in all succeeding cases in this paper where a unidirectional hypothesis was tested, a one-tailed test was employed and is indicated by the notation 1/2p.) Similar results occurred for analyses performed on HR and FAR after the froot transformation had been applied. Furthermore, separate analyses of variance conducted on each condition yielded significant effects of optimal beta level for HR and FAR in all conditions, both with and without the froot transformation.

In conirast, the effect of optimal beta level on d' was not significant in the overall analysis involving all conditions $[F(1,50)=1.1, \mathrm{MSe}=.2, \mathrm{p}=.3]$, nor in any of the analyses conducted on each condition separately. The interaction of condition and optimal beta level was also not significant $[F(4,50)<1]$. It seems unlikely that the nonsignificance of optimal beta level on $\mathrm{d}^{\prime}$ is attributable merely to a lack of power of the statistical test, since 60 subjects were employed for the overall analysis, which was not significant for $\mathrm{d}^{\prime}$, whereas only 12 subjects were employed in each individual condition, and optimal beta level was significant for HR and FAR for each condition considered separately. Furthermore, the overall analysis of $d^{\prime}$ had enough power to reveal a strong main effect of condition $[\mathrm{F}(4,50)=12.1, \mathrm{MSe}=.9, \mathrm{p}<.001]$, reflecting the difference between the no-memory condition and the other four conditions, as well as a significant main effect of ordinal block position $[F(3,150)=4.8$, $\mathrm{MSe}=.3, \mathrm{p}=.003]$ and a significant three-way interaction of Condition by Optimal Beta Level by Ordinal Block Position $[\mathrm{F}(12,150)=2.4, \mathrm{MSe}=.2, \mathrm{p}=.007]$, effects that are not readily interpretable. Moreover, although there does appear to be a trend in the data suggesting that $\mathrm{d}^{\prime}$ was larger when optimal beta was 1 than when optimal beta was 3 , only 6 of the 12 subjects in the payoff condition and 30 of the 48 subjects in the other conditions showed this effect. Nevertheless, it would be valuable to determine whether $\mathrm{d}^{\prime}$ remains insensitive to priors and payoffs over a larger range of HRs and FARs. However, because of the conservative cutoff placement typically exhibited by subjects, it would be difficult to obtain a substantially larger range of HRs and FARs by varying only priors or payoffs. In any event, these results suggest that $\mathrm{d}^{\prime}$ was not sensitive to changes in priors or payoffs.

On the other hand, $p_{1}$ and $p_{2}$ were sensitive to changes in priors. Specifically, the effect of optimal beta level was significant in the overall analyses involving all conditions for both indices $\left[\mathrm{p}_{1}: \mathrm{F}(1,50)=12.0, \mathrm{MSe}=\right.$ $\left..06, \mathrm{p}=.001 ; \mathrm{p}_{2}: \mathrm{F}(1,50)=6.5, \mathrm{MSe}=.02, \mathrm{p}=.01\right]$, and the interaction of condition and optimal beta level was not significant for either index $\left[\mathrm{p}_{1}: F(4,50)=1.2\right.$, $\left.\mathrm{MSe}=.06, \mathrm{p}=.3 ; \mathrm{p}_{2}: \mathrm{F}(4,50)=1.4, \mathrm{MSe}=.02, \mathrm{p}=.2\right]$. Similar results were found for overall analyses performed on $p_{1}$ and $p_{2}$ after the froot transformation had been applied. For $p_{1}$ the effect of optimal beta level was significant in separate analyses for each of the recognition memory with priors conditions (standard, tally, and no feedback) but not in the analyses for the other conditions. For $p_{2}$ the effect of optimal beta level was significant in the no-feedback condition but was not significant in any of the other conditions. The same pattern of results was found for the transformed values of $p_{1}$ and $p_{2}$. Note that although the interaction of condition and optimal beta level was not significant for $\mathrm{p}_{1}$ and $\mathrm{p}_{2}$, the effect of optimal beta level was smaller and in the opposite direction for the payoff condition than for the conditions with changes in priors. Hence 
it is possible that $p_{1}$ and $p_{2}$ are not as sensitive to changes in payoffs as they are to changes in priors.

To summarize, $p_{1}$ and $p_{2}$, but not $d^{\prime}$, were sensitive to changes in prior probability levels in the present experiment, with $p_{1}$ somewhat more sensitive than $p_{2}$.

\section{Cutoff Location}

Effects on beta. Figure 4 presents the mean values of beta averaged across subjects and blocks as a function of optimal beta level and condition. Overall, beta shifted with changes in priors and payoffs, but the shifts were considerably smalıer than those prescribed by the normative model, according to which the subject should switch from a beta of 1 to a beta of 3 . Hence conservative cutoff placement was obtained in this experiment. An analysis of variance revealed a strong main effect of optimal beta level $[F(1,50)=16.5$, $\mathrm{MSe}=1.1,1 / 2 \mathrm{p}<.001]$ and no interaction of condition and optimal beta level $[F(4,50)<1]$. Separate analyses of variance conducted with each condition revealed significant effects of optimal beta level only for the three recognition memory with priors conditions (standard, no feedback, and tally). Similar results were found for an analysis conducted on values of beta after a logarithmic transformation had been applied, but in this case, in addition, in the overall analysis the main effect of condition was significant $[F(4,50)=3.9$, $\mathrm{MSe}=.6, \mathrm{p}=.008]$ (which can be understood, at least in part, by the lower values of $\log$ beta in the nomemory condition; see below). Furthermore, in the separate analyses for log beta, optimal beta level was significant for the payoff condition as well as for the three recognition memory with priors conditions. Moreover, in an unpublished replication of the payoff condition, optimal beta level was significant for both beta and log beta.

The shifts in beta with changes in priors in recognition inemory appear to be inconsistent with the results of Healy and Jones (1975) and Healy and Kubovy (1977). In order to clarify the apparent discrepancy between the present results and the comparable results from Healy and Kubovy (1977), an analysis was conducted to compare the standard condition to the analogous condition in the study by Healy and Kubovy (1977, Experiment 2). The analysis revealed a significant main effect of prior probability level $[F(1,20)=5.3$, $\mathrm{MSe}=.2,1 / 2 \mathrm{p}=.02]$, but neither a significant main effect of experiment nor significant interactions with experiment. When a logarithmic transformation was applied to these data, neither the main effect of prior probability, nor the main effect of experiment, nor any interactions with experiment were significant. These results suggest that the effect of prior probability level on beta in recognition memory is no greater in the present experiment than in the experiment by Healy and Kubovy (1977), but rather that the data are more stable in the present experiment than in the earlier one.

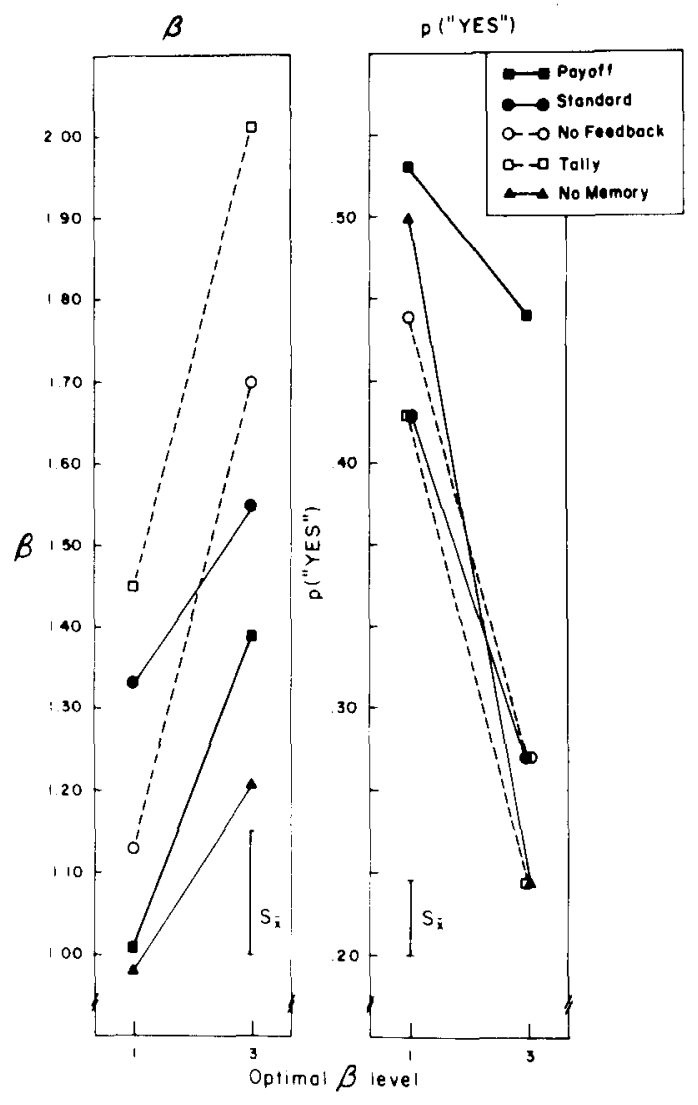

Figure 4. Mean values of beta (left panel) and p("yes") (right panel) as a function of condition and optimal beta level. The standard error of the means for each index, as determined by an analysis of variance, is indicated at the bottom of the appropriate panel.

In order to test the memory-load hypothesis, according to which the effect of priors on beta should be smaller than the effect of payoffs on beta, two analyses of variance were conducted to compare the standard and payoff conditions, one with untransformed values of beta and one with values of log beta. For both analyses, the main effect of optimal beta level was significant, but neither analysis revealed a significant effect of condition or a significant interaction of condition and optimal beta level. These results are inconsistent with the memory-load hypothesis. In addition, these results pose some difficulty for any explanation of conservative cutoff placement that deals exclusively with how the subject handles payoffs or prior probabilities, such as an explanation in terms of prior probability matching. The similar magnitudes of shifts in observed values of beta with changes in optimal beta levels for payoffs and priors are purely coincidental according to these explanations.

Despite the significant shifts in beta found in the standard condition, it is still of interest to determine the effect of trial-by-trial feedback information on those shifts, in order to provide a more rigorous test of the memory-load hypothesis. In fact, the shifts in cutoff 
appear somewhat larger in both the no-feedback and tally conditions than in the standard condition, contrary to the memory-load hypothesis, according to which the shifts should be larger only in the tally condition. Two analyses of variance were conducted to compare the standard condition to the tally condition and to the no-feedback condition. Neither analysis revealed a significant main effect of condition or a significant interaction of prior probability level and condition. An analogous pair of analyses conducted on values of $\log$ beta yielded the same pattern of results. These analyses suggest that the amount of feedback does not affect the extent of shifts in observed beta with changes in prior probability level in recognition memory, contrary to the memory-load hypothesis.

Another test of the memory-load hypothesis can be made by comparing the standard condition and the no-memory condition, although a conparison of observed values of beta is suspect, since discriminability was found to be essentially zero in the no-memory condition. With $\mathrm{d}^{\prime}$ exactly 0 , beta will necessarily be exactly 1 . In fact, although an analysis of variance performed on observed values of beta for the standard and no-memory conditions did not reveal a significant main effect of condition or a significant interaction of condition and prior probability level, an analysis performed on values of $\log$ beta did reveal a significant main effect of condition. As we will discuss below, a more appropriate comparison of cutoff locations in the standard and no-memory conditions involves the proportion of "yes" responses, p("yes").

Effects on p("yes"). The index p("yes") is a modelfree measure of cutoff location, and for that reason may be preferable to beta, or to any other index of the criterion (Healy \& Jones, 1973). However, a direct comparison of the extent of shifts in p("yes") as a function of changes in optimal beta levels is not a meaningful comparison when conditions with changes in priors are compared to conditions with changes in payoffs, since $p$ ("yes") will decrease with decreasing prior probability levels even if the subject holds beta constant, but a similar decrease will not be found with changes only in the payoff matrix. Figure 4 presents the rinean values of $p$ ("yes") averaged across subjects and blocks as a function of condition and optimal beta level. As expected on the basis of the argument given above, p("yes") was larger in the payoff condition than in the four conditions with changes in priors (standard, tally, no feedback, and no memory) $[\mathrm{F}(4,50)=6.7, \mathrm{MSe}=.06$, $\mathrm{p}<.001]$, and the difference between these two sets of conditions was especially marked for the higher level of optimal beta $[F(4,50)=3.7, \mathrm{MSe}=.04, \mathrm{p}=.01]$. It is also interesting to note that in the four conditions with changes in prior probability levels, p("yes") matched quite closely the proportions of old items in the test- -.50 when optimal beta was 1 and .25 when optimal beta was 3 . In other words, there is some evidence for a probability-matching strategy. Such a strategy cannot, however, account for any shifts in p("yes") when payoffs change.

In order to confirm that subjects do shift their cutoff locations with changes in payoffs in recognition memory, an analysis was conducted of p("yes") in the payoff condition. Recall that optimal beta level had a significant effect on $\log$ beta (but not on beta) in the payoff condition. Likewise, optimal beta level had a significant effect on p("yes") in the payoff condition. A similar result was found for an analysis conducted on $p$ ("yes") after the froot transformation had been applied. These analyses confirm that subjects do shift their cutoff locations with changes in payoffs in recognition memory.

The question remains whether subjects will show similar shifts in cutoff location with changes in prior probabilities in the no-memory condition as in the standard condition. This question, which is crucial to the memory-load hypothesis, cannot be answered definitively on the basis of an analysis of changes in observed values of beta, as discussed above. The question can be answered, however, by considering p("yes"). An analysis of variance on $p$ ("yes") in the standard condition and the no-memory condition was conducted. Although the shift appears to be somewhat greater in the no-memory condition than in the standard condition, the main effect of condition and the interaction of condition and prior probability level were not significant. The same pattern of results was found for an analysis conducted on $p$ ("yes") after the froot transformation had been applied. These analyses suggest that subjects do shift their cutoffs in the no-memory condition to the same extent that they do in a comparable recognition memory task, which is inconsistent with the memory-load hypothesis and with Kubovy's (1977) distribution-misconception explanation of conservative cutoff placement.

To summarize, the results of this experiment indicate roughly equal shifts in cutoff location with changes in priors and payoffs. In addition, shifts in cutoff location were not affected by the amount of feedback provided or the presence of a memory load. We have, therefore, no support for the memory-load hypothesis, for Kubovy's (1977) distribution-misconception explanation of conservative cutoff placement, or for any explanation of conservative cutoff placement dealing exclusively with payoffs or priors, such as the probability-matching explanation. Subjects do appear, however, to match $p$ ("yes") to the prior probability of an old item when payoffs are symmetric.

\section{CONCLUSIONS}

\section{Indices of Performance}

Both $p_{1}$ and $p_{2}$, unlike $d^{\prime}$, are not truly criterion-free indices of recognition performance, since they are sensitive to changes in prior probability levels in recognition memory tasks. 
The sensitivity of $p_{1}$ and $p_{2}$ to changes in prior probabilities found here provides further evidence against the simple threshold models. Thus, $d^{\prime}$ remains the preferred index of recognition memory performance, and $p_{1}$ and $p_{2}$ should not be employed as indices of memory performance, since they are not bias free and hence do not adequately correct for guessing.

The following question remains: Which index of performance should be used to compare performance levels on recall and recognition tests, since $\mathrm{d}^{\prime}$ is not useful for that purpose? One solution would be for the investigator to use a forced-choice procedure, rather than a yes-no procedure, in the recognition test, so that the subject would select from a fixed number of response alternatives presented on a given trial. A similar procedure could be used for recall by prohibiting omissions. This solution would bypass the criterion problem, since in both cases, presumably, the strongest response would be made. However, this method would not correct for guessing, which would still be possible in both the recall and recognition tasks. Hence, this method would not provide an accurate estimate of the amount of information retained in memory. If the probabilities of responding correctly when the subject has no information differ in the recall and recognition tests, as they most certainly would, the measures of recall and recognition performance would not be strictly comparable. Researchers have typically used threshold models to derive corrections for guessing in recall (see, e.g., Sperling \& Melchner, 1976). The evidence provided here against the threshold corrections in recognition should also throw some doubt on the use of such corrections in recall. However, there does not seem to be an alternative criterion-free performance index for recall.

\section{Cutoff Location}

A "memory-load" hypothesis was put forth to explain the relative insensitivity of subjects to prior probabilities in recognition memory tasks (Healy \& Jones, 1975; Healy \& Kubovy, 1977). According to this hypothesis, the recognition memory task imposes memory requirements that are incompatible with the subject's effective use of prior probability information. Three types of evidence were marshalled against this hypothesis. First, the amount of feedback provided to the subjects did not affect the size of the shift in cutoff with changes in priors. Second, the size of the shift in cutoff with changes in priors was roughly equal to the size of the shift in cutoff with changes in payoffs. Third, the cutoff shift was not significantly smaller in the recognition memory task than in a task in which no memory was required. This last fact suggests that the conservative cutoff placement found in recognition memory is not a result of the peculiar demands of the recognition memory task. Especially in light of this conclusion, it seems reasonable to make use of these results to evaluate the alternative explanations that have been proposed more generally for conservative cutoff placement in probabilistic categorization.

One explanation for conservative cutoff placement is the distribution-misconception notion recently proposed by Kubovy (1977) on the basis of data from a numerical decision task. According to this notion, subjects have a systematic misconception of the underlying stimulus distributions. Such an explanation cannot account for conservative cutoff placement in the no-memory condition, since the subjects should essentially ignore the stimulus distributions in that condition. Nevertheless, conservative cutoff placement was observed in the nomemory condition, and the magnitudes of the shifts in cutoff in that condition were not significantly different from those in the comparable memory condition.

Other explanations for conservative cutoff placement are specific to situations with changing priors or changing payoffs. One such explanation, which is specific to changing priors, is in terms of prior probability matching. In fact, in the present experiment, we did find that the values of $p$ ("yes") were relatively closely matched to the prior probabilities of an old item. However, the probability-matching strategy cannot account for the significant change in cutoff location [and, correspondingly, in p("yes")] found in this study when payoffs varied. Another explanation is required for conservative cutoff placement in the case of changing payoffs, and the observed similar cutoff locations when payoffs and priors varied can only be seen as coincidental. We are presently conducting further studies to determine whether cutoff locations for changing priors and changing payoffs will continue to coincide when a larger range of priors and payoffs is considered. For now, the probability-matching strategy cannot be ruled out definitively. If, however, future studies do continue to indicate a coincidence of cutoff locations for payoffs and priors that specify the same levels of optimal beta, there would be support for the notion that subjects systematically miscalculate where to place the cutoff on the observation continuum given the correct calculation of optimal beta.

\section{REFERENCE NOTE}

1. Egan, J. P. Recognition memory and the operating characteristic. (Tech. Rep. No. AFCRC-TN-58-51, AD-152650) Indiana University, Hearing and Communication Laboratory, 1958.

\section{REFERENCES}

ANDERson, J. R. Language, memory, and thought. Hillsdale, N.J: Erlbaum, 1976.

Craig, A. Signal recognition and the probability-matching decision rule. Perception \& Psychophysics, 1976, 20, 157.162.

Creelman, C. D., \& Donaldson, W. ROC curves for discrimination of linear extent. Journal of Experimental Psychology, 1968, 77, 514-516.

Dorfman, D. D. Probability matching in signal detection. Psychonomic Science, 1969, 17, 103. 
Estes, W. K., \& DaPolito, F. Independent variation of information storage and retrieval processes in paired-associate learning. Journal of Experimental Psychology, 1967, 75, 18-26.

Galanter, E. Psychological decision mechanisms and perception. In E. C. Carterette \& M. P. Friedman (Eds.), Handbook of perception (Vol. 2). New York: Academic Press, 1974.

Green, D. M., \& Moses, F. L. On the equivalence of two recognition measures of short-term memory. Psychological Bulletin, 1966, 66, 228-234.

Healy, A. F., \& Jones, C. Criterion shifts in recall. Psychological Bulletin, 1973, 79, 335-340.

Healy, A. F., \& Jones, C. Can subjects maintain a constant criterion in a memory task? Memory \& Cognition, 1975, 3, 233-238.

Healy, A. F., \& Kubovy, M. A comparison of recognition memory to numerical decision: How prior probabilities af fect cutoff location. Memory \& Cognition, 1977, 5, 3-9.

KInTSCH, W. Models for free recall and recognition. In D. A. Norman (Ed.), Models of human memory. New York: Academic Press, 1970.

Krantz, D. H. Threshold theories of signal detection. Psychological Review, 1969, 76, 308-324.

Kunovy, M. A possible basis for conservatism in signal detection and probabilistic categorization tasks. Perception \& Psychophysics, 1977, 22, 277-281.

Kubovy, M., \& Healy, A. F. The decision rule in probabilistic categorization: What it is and how it is learned. Journal of Experimental Psychology: General, 1977, 106, 427-446.

Kubovy, M., \& Healy, A. F. Process models of probabilistic categorization decisions. In T. Wallsten (Ed.), Cognitive processes in choice and decision behavior. Hillsdale, N.J: Erlbaum, in press.

LUCE, R. D. A threshold theory for simple detection experiments. Psychological Review, 1963, 70, 61-79.

Mosteller, F., \& Tukey, J. W. Data analysis and regression: A second course in statistics. Reading, Mass: Addison-Wesley, 1977.

MURdock, B. B., JR. Signal-detection theory and short-term memory. Journal of Experimental Psychology, 1965, 70, 443-447.

MURDOCK, B. B., JR. Human memory: Theory and data. Potomac, Md: Erlbaum, 1974

PARKs, T. E. Signal-detectability theory of recognition memory performance. Psychological Review, 1966, 73, 44-58.
Sperling, G., \& Melchner, M. J. Estimating item and order information. Journal of Mathematical Psychology, 1976. 13, 192-213.

Swets, J. A., Tanner, W. P., JR., \& Birdsall, T. G. Decision processes in perception. Psychological Review, 1961, 68, 301-340.

Thomas, E. A. C., \& Legge, D. Probability matching as a basis for detection and recognition decisions. Psychological Review, 1970, 77, 65-72.

Tulving, E., \& Thomson, D. M. Encoding specificity and retrieval processes in episodic memory. Psychological Review, 1973, 80, 352-373.

Ulehla, Z. J. Optimality of perceptual decision criteria. Journal of Experimental Psychology, 1966, 71, 564-569.

\section{NOTES}

1. Note, however, that in general, the two formulas lead to different estimates of $p$ (unless FAR $=0$ ), with $p_{1}$ greater than $p_{2}$. The present study will not allow us to determine which index provides the more accurate estimate of the amount of information retained in memory. It should also be noted that, as in recognition, there is a problem of correcting for guessing in recall, which we will not be concerned with in this paper. Such a problem is most clear in a recall situation with a limited number of response alternatives or in a cued recall situation in which the cues are associated with the correct responses. In both these situations, the subject may be able to guess correctly the to-berecalled item even when there is no memory for it. Hence, some correction for guessing is also necessary for recall in order to provide an accurate estimate of the amount of information retained in memory.

2. The numbers shown in a given block of trials were selected in a pseudorandom manner without replacement from the population of 400 different five-digit numbers employed by Healy and Kubovy (1977, Experiment 2, recognition memory condition). The details concerning the construction of this population should not be relevant to the present experiment and hence will not be reviewed here. These details are available, however, from the report by Healy and Kubovy (1977).

(Received for publication August 9, 1978; accepted August 21, 1978.) 